\title{
Pulmonary Arterial Hypertension Management - A New Approach For a Rare Disease
}

\author{
Jean-Luc Vachiery \\ Pulmonary Vascular Diseases Clinic, Department of Cardiology, Cliniques Universitaires de Bruxelles, Hôpital Erasme, Brussels, Belgium
}

DOl: http://doi.org/10.17925/ERPD.2016.02.02.50

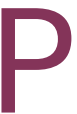
ulmonary Arterial Hypertension is a rare, deadly and incurable condition for which management guidelines have recently been revised. Important and remarkable changes include a new strategy-oriented treatment algorithm, based on a risk-stratification approach. Combination therapy is recommended as soon as diagnosis is established for all patients, with a parenteral prostacyclin for the most severe cases. The uncommon nature of the disease and the management complexity require that care is delivered by expert specialised centers.

\section{Keywords}

Pulmonary arterial hypertension, guidelines, combination therapy, expert, risk stratification, pulmonary hypertension

Disclosure: Jean-Luc Vachiery has nothing to disclose in relation to this paper. This article is a short opinion piece and has not been submitted to external peer reviewers. No funding was received for the publication of this article.

open Access: This article is published under the Creative Commons Attribution Noncommercial License which permits any non-commercial use, distribution, adaptation and reproduction provided the original author(s) and source are given appropriate credit.

Received: 8 July 2016

Published Online: 12 August 2016

Citation: European Respiratory \& Pulmonary Diseases, 2016;2(2):50-1

Corresponding Author: Jean-Luc Vachiery, Department of Cardiology, CUB - Hopital Erasme, 808 Route de Lennik 1070 - Brussels, Belgium.

E: jeanluc.vachiery@erasme.ulb.ac.be
Pulmonary arterial hypertension (PAH) is a rare, deadly and incurable condition. ${ }^{1}$ With a five-year survival rate below $60 \%$, the outcome of this disease is not any better than that of many cancers. ${ }^{12}$ However, numerous treatment options are currently available: more than 20 randomised controlled trials (RCTs) have been conducted over the past 10 years, allowing for the approval of 10 PAH-specific drugs by regulatory agencies worldwide. ${ }^{1,2}$ In addition, the recent completion of four event-driven RCTs geared the management of PAH towards a new era. ${ }^{3-6} \mathrm{PAH}$ is therefore one of the very few rare conditions for which international practice guidelines were established more than 10 years ago. In 2015, the European Society of Cardiology and the European Respiratory Society (together with the International Society for Heart and Lung Transplantation) joined forces to revise previous guidelines and present the most recent standards for the management of PAH. ${ }^{1}$ Three major changes warrant special attention.

The first change is risk stratification, which has been proposed as the gatekeeper to the treatment algorithm. Such an essential exercise requires careful assessment and allows clinicians to stratify individual patients based on three factors:

1. Clinical, which includes New York Heart Association (NYHA) functional class and signs of right heart failure;

2. Exercise capacity, using either the six-minute walking distance (6MWD) test or the more sophisticated cardiopulmonary exercise test; and

3. Right ventricular function, with special attention to invasive haemodynamics.

Up to 14 meaningful, easily retrievable variables are considered in this analysis to establish whether the one-year mortality risk is low $(<5 \%)$, intermediate $(5-10 \%)$ or high $(>10 \%)$. This evaluation is recommended both at baseline (in treatment-naïve patients) and during follow up (to demonstrate inadequate clinical response).

The second change is a new strategy-oriented treatment algorithm, moving away from a more traditional drug-driven standard. This algorithm reflects an important paradigm shift in the management of PAH. There is indeed little question that combination therapy is effective in PAH. Recently published RCTs demonstrate that adding a second agent improves exercise capacity (by 6MWD) in patients receiving riociguat? (a soluble guanalyte cyclase stimulator, GCs) or macitentan $^{3}$ (an endothelin receptor antagonist [ERA]), and delays clinical worsening in patients receiving macitentan ${ }^{3}$ or selexipag (a prostacyclin receptor agonist). In newly diagnosed patients, combination therapy with ambrisentan (an ERA) and tadalafil (a phosphodiesterase type 5 inhibitor, PDE5i) delays time to clinical worsening compared with monotherapy with either compound. ${ }^{4}$ Finally, patients presenting severe, high-risk PAH appear to benefit from a combination of parenteral prostacyclin and oral agents. ${ }^{1}$ Therefore, a sound strategy would favour initial combination therapy in patients with intermediate or high risk, the latter including a parenteral prostacyclin. 
Finally, the management of PAH should be driven by expert specialised centres. PAH is a life-threatening, disabling and complex disease. The burden for patients is high, because it also leads to isolation, psychosocial impairment and high economic cost. PAH affects patients, families, caregivers and healthcare providers in many different ways. It is therefore the mission of such PAH expert centres to provide holistic care in a multidisciplinary, multiprofessional way. This includes a requirement for networking and establishing links to other services and procedures for management, which must include those leading to lung transplantation programmes. In a PAH clinic, the presence of specialised nurses is of critical importance as it is known to be one of the key drivers of success. Finally, an important part of the core missions of a PAH centre is education, research and collaboration. Without such strong bonds, it is likely that the management of PAH would still be in the dark ages.
Despite these significant advances, there is still a large potential for improvement. PAH is a complex disorder involving many pathobiological, molecular and genetic pathways, most of which are not completely understood. Only three of these pathways have led to approval of various agents (ERA, GCS/PDE5i and prostacyclins) and more are currently under investigation. Using the currently available resources at their best is also important, for example, by exploring switching strategies or developing better tools for monitoring to help PAH teams in the decision-making process. Properly conducting RCTs, including redefining endpoints and addressing ethical issues, is one of the numerous missions ahead of us. All these missions will have to be accomplished in the context of economical constraints and cuts in healthcare expenditures worldwide. This will require tremendous effort from all stakeholders (from basic researchers to clinicians, from industry to healthcare regulatory agencies), which must include the patient's representatives. $\square$
1. Galiè N, Humbert M, Vachiery JL, et al., 2015 ESC/ERS Guidelines for the diagnosis and treatment of pulmonary hypertension Eur Heart J, 2016:37:67-119.

2. Kanwar $\mathrm{M}$, Thenappan T Vachiéry JL, Update in treatment options in pulmonary hypertension, $J$ Heart Lung Transplant, 2016;35:695-703.

3. Pulido T, Adzerikho I, Channick RN, et al., Macitentan and morbidity and mortality in pulmonary arterial hypertension N Engl J Med, 2013;369:809-818.

4. Galie N, Barbera JA, Frost A, et al., AMBITION: A randomised, multicenter study of first-line ambrisentan and tadalafil combination therapy in subjects with pulmonary arteria combination therapy in subjects with pulmonary
hypertension (PAH), Eur Respir J, 2014;44:2916. 5. Sitbon O, Channick R, Chin K, et al., Selexipag for the Treatment of
Pulmonary Arterial Hypertension, N Eng/ J Med, 2015;373:2522-33. 6. McLaughlin V, Channick RN, Ghofrani HA, et al., Bosentan added to sildenafil therapy in patients with pulmonary arteria added to sildenafll therapy in patients with pur

7. Ghofrani HA, Galie N, Grimminger F, et al, Riociguat for the treatment of pulmonary arterial hypertension, N Engl I Med, 2013;369:330-40 